\title{
Rheological properties, textural properties, and storage stability of palm kernel-based diacylglycerol-en riched mayonnaise
}

\begin{abstract}
The effect of replacement of soybean oil (SBO) with $0,5,10,15$, and $20 \%$ palm kernel based-diacylglycerol (PKDG) which were referred to as SD1000, SD9505, SD9010, SD8515, and SD8020 formulations, respectively, on the rheological properties, textural properties, and storage stability of mayonnaise were evaluated in this study. Emulsion stability results demonstrated no significant differences (P®[0.05) among all formulations except SD8020 which exhibited diminished emulsion stability. In terms of textural properties, both SD9010 and SD8020 showed closer textural characteristics compared with control. Microstructure evaluation of all mayonnaise formulations revealed significant increases in oil droplet diameter in tandem with increasing PKDG concentration. For rheological properties, all mayonnaise formulated displayed similar gel-like behavior with greater storage modulus $\left(\mathrm{G}^{\prime}\right)$ than loss modulus (Gò) and loss tangent (tan ŭ) less than 0.3 . Results indicated that up to $10 \%$ PKDG was suitable to be incorporated in healthy mayonnaise formulation. Both SD9505 and SD9010 were subsequently evaluated for their stability throughout a 2-month storage period. All mayonnaise displayed satisfying stability during storage with gradual increment in peroxide value, free fatty acid level, and G'Gò crossover value except control. Additionally, an insignificant change in viscoelastic properties (P $\mathrm{P}$ (ID.05) was observed after storage for all formulations examined. Practical applications: This present work has three main practical applications: (i) enable the incorporation of PKDG as a functional ingredient into a mayonnaise system; (ii) provide knowledge and understanding of the physicochemical, rheological, and textural properties of PKDG-enriched mayonnaise; and (iii) give insight into the storage stability of PKDG-incorporated mayonnaise throughout the 2-month storage period.
\end{abstract}

Keyword: Diacylglycerol; Mayonnaise; Palm kernel; Rheological properties; Storage stability 